\title{
200004
}

4-9 July $2004 \cdot$ Phosphorus Chemistry $•$ Birmingham, United Kingdom 16th International Conference on Phosphorus Chemistry (ICPC 16)

Prof. Pascal Metivier, Rhodia, R\&D for Phosphorous and Performance Derivatives, Oak House, reeds Crescent, Watford, WD24 4QP, UK, Tel.: +44 1923 485609, E-mail: pascal.metivier@eu.rhodia.com

4-9 July $2004 \cdot$ Macromolecules $•$ Paris, France 40th International Symposium on Macromolecules_IUPAC World Polymer Congress (MACRO 2004) Prof. Jean-Pierre Vairon, Université Pierre et Marie Curie, Laboratoire de Chimie des Polymères, Case 185, 4 Place Jussieu, F-75252 Paris Cédex 05, France, Tel: +33 1442750 45, Fax: +33 144277089 , E-mail: macro04@ccr.jussieu.fr

11-15 July 2004 • Polymer Biomaterials • Prague, Czech Republic 43rd PMM Microsymposium: Polymer Biomaterials: Biomimetic and Bioanalogous Systems Drahomir Vyprachticky, Institute of Macromolecular Chemistry, Heyrovskeho nam. 2, CZ-162 06 Praha 6, Czech Republic, Tel.: +420 296809 332, Fax: +420 296809 410, E-mail: sympo@imc.cas.cz

17-22 July $2004 \cdot$ Photochemistry • Granada, Spain 20th IUPAC Symposium on Photochemistry Prof. Dr. Miguel A. Miranda, Departamento de Química/Instituto de Tecnologia Química UPV-CSIC, Universidad Politecnica de Valencia, Avenida de los Naranjos, s/n, E-46022 Valencia, Spain, Tel: + 34 963877807, Fax: + 34 963877809, E-mail: mmiranda@qim.upv.es

18-21 July 2004 • Chemical Sciences in Changing Times • Belgrade, Serbia and Montenegro 4th International Conference of the Chemical Societies of the South-Eastern European Countries Prof. Ivanka Popovic, Belgrade University, Faculty of Technology and Metallurgy, Karnegijeva 4, 11000 Belgrade, Serbia and Montenegro, Tel.: +381 11337 0478, Fax: +381 11337 0473, E-mail: icosecs@elab.tmf.bg.ac.yu

18-23 July $2004 \cdot$ Coordination Chemistry • Merida, Yucatan, Mexico 36th International Conference on Coordination Chemistry Prof. Norah Barba-Behrens, Dept. de Química Inorgánica, Universidad Nacional Autónoma de México, Ciudad Universitaria, Coyoacán, México, D. F., 04510, México, Tel./Fax: +52(55)5622-3810, E-mail: norah@servidor.unam.mx

18-23 July 2004 • Polymers and Organic Chemistry • Prague, Czech Republic 11th International Conference on Polymers and Organic Chemistry 2004 (POC '04)

Dr. Karel Jerabek, Institute of Chemical Process Fundamentals, Rozvojova 135, 16502 Prague 6, Czech Republic, Tel.: +420 220390 332, Fax: + 420220920 661, E-mail: kjer@icpf.cas.cz

23-27 July $2004 \cdot$ Carbohydrates • Glasgow, United Kingdom

22nd International Carbohydrate Symposium

Prof. E. Hounsell, School of Biological and Chemical Sciences, Birkbeck University of London, Malet St., London WC1E7HX, UK, Tel.: + 44207631 6238, E-mail: e.hounsell@bbk.ac.uk

25-29 July $2004 \cdot$ Solubility Phenomena • Aveiro, Portugal 11th International Symposium on Solubility Phenomena, Including Related Equilibrium Processes (11th ISSP) Prof. Clara Magalhaes, Department of Chemistry, University of Aveiro, P-3810-193 Aveiro, Portugal, Tel.: +351 234 401518, Fax: +351 234 370084, E-mail: issp@dq.ua.pt

25-30 July $2004 \cdot$ Organometallic Chemistry • Vancouver, Canada 21st International Conference on Organometallic Chemistry (ICOMC)

21st ICOMC Secretariat, Conferences \& Accomodation at UBC, 5961 Student Union Boulevard, Vancouver, BC, Canada V6T 2C9, Tel.: +1 604 822-1050, Fax: +1 604 822-1069, E-mail: registration@housing.ubc.ca

1-6 August $2004 \cdot$ Organic Synthesis • Nagoya, Japan 15th International Conference on Organic Synthesis (ICOS-15) (see poster on inside back cover) Prof. Minoru Isobe, ICOS15 Secretariat, c/o International Communications Specialists, Inc., Sabo Kaikan-bekkan, 2-7-4 Hirakawa-cho, Chiyoda-ku, Tokyo 102-8646 Japan, Tel: +81 33263 6474,

Fax: +81 33263 7537, E-mail: icos@ics-inc.co.jp

2-7 August $2004 \cdot$ Chemistry in Africa • Arusha, Tanzania 9th International Chemistry Conference in Africa-Chemistry Towards Disease and Poverty Eradication Dr. G. S. Mhinzi, University of Dar es Salaam, Chemistry Department, PO Box 35061, Dar es Salaam, Tanzania, Tel./Fax: +255 22 2410038, E-mail: mhinzi@chem.udsm.ac.tz 


\section{Mark Your Calendar}

3-8 August 2004 • Chemical Education • Istanbul, Turkey 18th International Conference on Chemical Education (18th ICCE)

Prof. Dr. Mustafa L. Berkem, Chairman, Marmara University, Ataturk Faculty of Education, TR- 81040 GoztepeIstanbul, Turkey, Tel: +90 2163459090/231, Fax: +90 2163388060, E-mail: icce2004@marmara.edu.tr

15-19 August $2004 \cdot$ Polymers $•$ Bethesda, Maryland, USA

Polymer Networks 2004

Dr. F. Horkay, Section on Tissue Biophysics and Biomimetics, National Institutes of Health, Bldg. 13, Room 3W16E, 13 South Drive, Bethesda, MD 20892, USA, Tel: +1 301435 7229, Fax: +1 301435 5035, E-mail: horkay@helix.nih.gov

15-20 August $2004 \cdot$ Physical Organic Chemistry • Shanghai, China

17th IUPAC Conference on Physical Organic Chemistry (ICPOC-17)

Prof. Guo-Zhen Ji, Shanghai Institute of Organic Chemistry, Chinese Academy of Sciences, 354 Fenglin Road, Shanghai 200032, China, Tel: +86 21-64163300, Fax: +86 21-64166128, E-mail: jigz@pub.sioc.ac.cn

17-21 August 2004 • Chemical Thermodynamics • Beijing, China 18th IUPAC Conference on Chemical Thermodynamics

Prof. Haike Yan, Chairman, 18th ICCT c/o Chinese Chemical Society, PO Box 2709, Beijing, 100080, China, Tel.: +8610 62568157, +86 10 62564020, Fax: +86 10 62568157, E-mail: qiuxb@infoc3.icas.ac.cn

20-25 August $2004 \cdot$ Heteroatom Chemistry $•$ Shanghai, China 7th International Conference on Heteroatom Chemistry (ICHAC-7)

Prof. Lin-xin Dai, ICHAC-7, c/o Shanghai Institute of Organic Chemistry, Chinese Academy of Sciences, 354 Fenglin Road, Shanghai 200032, China, Tel: +86 21 64163300- 3405, Fax: +86 21 64166128, E-mail: ICHAC@pub.sioc.ac.cn

22-28 August $2004 \cdot$ Biological Polyesters • Beijing, China International Symposium on Biological Polyesters (ISBP 2004)

Prof. George Guo-Qiang Chen, Department of Biological Sciences \& Biotechnology, Tsinghua University, 100084 Beijing, China, Tel.: +86 10 62794217, Fax: +86 10 62794217, E-mail: chengq@mail.tsinghua.edu.cn

3-5 September 2004 • Chemistry of Vanadium • Szeged, Hungary 4th International Symposium on Chemistry and Biological Chemistry of Vanadium Prof. Tamas Kiss, University of Szeged, Department of Inorganic and Analytical Chemistry, PO Box 440, H-6701 Szeged, Hungary, Tel.: +36 62 544337, Fax: +36 62 420505, E-mail: tkiss@chem.u-szeged.hu

5-10 September 2004 • Analytical Chemistry • Salamanca, Spain European Conference on Analytical Chemistry-Euroanalysis XIII Prof. J. Hernández Méndez, Departamento de Química Analítica Nutrición y Bromatología, Universidad de Salamanca, E-37008 Salamanca, Spain, Tel./Fax: +34 923 294483, E-mail: jhm@usal.es

12-15 September 2004 • Heterocyclic Chemistry • Sopron, Hungary XXI European Colloquium on Heterocyclic Chemistry Prof. György Hajos, Chemical Research Center, Institute of Chemistry, H-1025 Budapest Pusztaszeri ut, Hungary, Tel.: +36 13257550 , Fax: +36 13257863 , E-mail: ghajos@chemres.hu

20-23 September 2004 • Soil Science • Wuhan, China Environmental Significance of Mineral-Organic Component-Microorganism Interactions in Terrestrial Systems Dr. P. M. Huang, Department of Soil Science, University of Saskatchewan, 51 Campus Drive, Saskatoon SK S7N 5A8 Canada, Tel.: +1 306966 6838, Fax: +1 306966 6881, E-mail: huangp@sask.usask.ca

7-8 October 2004 • Trace Elements in Food • Brussels, Belgium 2nd International Symposium on Trace Elements in Food (TEF 2) Dr. Michael Bickel, European Commission-Joint Research Centre, Institute for Reference Materials and Measurements, B-2440 Geel, Belgium, Tel.: +32 145717 34, Fax: +32 1457 17 87, E-mail: michael.bickel@cec.eu.int

17-22 October $2004 \cdot$ Biotechnology $•$ Santiago, Chile 12th International Biotechnology Symposium

Prof. Juan A. Asenjo, Centre for Biochemical Engineering and Biotechnology, University of Chile, Beauchef 861, Santiago, Chile, Tel.: +56 2 6784288, Fax: +56 2 6991084, E-mail: IBS2004@conicyt.cl

18-22 October 2004 • Chemical Engineering • Havana, Cuba Vth International Congress on Chemistry and Chemical Engineering

Prof. Alberto J. Núñez Sellés, Center of Pharmaceutical Chemistry, Sociedad Cubana de Quimica, Ave 21 \& 200, Rpto. Atabey, Apdo. 16042 Havana, CP 11600, Cuba, Tel.: + 537218 178, Fax: +53 7336 471,

E-mail: alberto@cgf.co.cu 
30 November-3 December 2004 • Agriculture • Jesenik, Czech Republic Chemistry for Agriculture

Dr. Adam Pawelczyk, Wroclaw University of Technology, Smoluchowskiego 25, 50-370 Wroclaw, Poland, Tel.: +48 (0) 71-3202930, Fax: +48 (0) 71 3203469, E-mail: adam.pawelczyk@pwr.wroc.pl

6-8 December 2004 • Fats, Oils, and Oilseeds Analysis and Production • Tunis, Tunisia IUPAC-AOCS Workshop

$<$ WwW.aocs.org/meetings/>

\section{5}

14-17 February 2005 • Crop Protection Chemistry in Latin America • San Jose, Costa Rica International Workshop on Crop Protection Chemistry in Latin America: Harmonized Approaches for Environmental Assessment and Regulation <www.iupac.org/symposia/2005/crop-protection-chemistry/index.html>

27 February-2 March 2005 • Heterocyclic Chemistry • Gainesville, Florida, USA 6th Florida Heterocyclic Conference Prof. Alan R. Katritzky, University of Florida, Dept. of Chemistry, Gainesville, FL 32611-7200, USA, Tel.: +1 352392 0554, Fax: +1 352392 9199, E-mail: katritzky@chem.ufl.edu

17-21 July $2005 \cdot$ Organometallic Chemistry • Geneva, Switzerland 13th International Symposium on Organometallic Chemistry Directed Towards Organic Synthesis (OMCOS-13), Prof. E. Peter Kündig, Department of Organic Chemistry, University of Geneva, 30 Quai Ernest Ansermet, $\mathrm{CH}$ 1211 Geneva 4, Switzerland, Tel.: +41 22379 6526, Fax: +41 22328 7396, E-mail: Peter.Kundig@chiorg.unige.ch

17-22 July $2005 \cdot$ Carotenoids • Edinburgh, Scotland 14th International Symposium on Carotenoids

Prof. Andrew J. Young, School of Biological and Earth Sciences, John Moores University, Byrom St. Liverpool L3 3AF, UK, Tel.: +44 1512312173 / 3575, Fax: + 44151207 3224, E-mail: a.j.young@livjm.ac.uk

13-21 August 2005 • IUPAC 43rd General Assembly • Beijing, China IUPAC Secretariat, Tel.: +1 919485 8700, Fax: +1 919485 8706, E-mail: secretariat@iupac.org

14-19 August 2005 • IUPAC 40th Congress-Innovation in Chemistry • Beijing, China Prof. Xibai Qiu, IUPAC-2005 Secretariat, c/o Chinese Chemical Society, PO Box 2709, Beijing 100080, China, Tel.: +86 (10) 62568157, Fax: +86 (10) 62568157, E-mail: qiuxb@iccas.ac.cn

11-15 September 2005 • Boron Chemistry • Sendai, Japan

12th International Meeting on Boron Chemistry

Prof. Yoshinori Yamamoto, Department of Chemistry, Graduate School of Science, Tohoku University, Sendai, Japan 980-8578, Tel.: +81 22217 6581, Fax: +81 22217 6784, E-mail: yoshi@yamamoto1.chem.tohoku.ac.jp

\section{Visas}

It is a condition of sponsorships that organizers of meetings under the auspices of IUPAC, in considering the locations of such meetings, should take all possible steps to ensure the freedom of all bona fide chemists from throughout the world to attend irrespective of race, religion, or political philosophy. IUPAC sponsorship implies that entry visas will be granted to all bona fide chemists provided application is made not less than three months in advance. If a visa is not granted one month before the meeting, the IUPAC Secretariat should be notified without delay by the applicant.

\section{How to Apply for IUPAC Sponsorship}

Conference organizers are invited to complete an Application for IUPAC Sponsorship (AIS) preferably 2 years and at least 12 months before the Conference. Further information on granting sponsorhsip is included in the AIS and is available upon request from the IUPAC Secretariat or online.

$<$ www.iupac.org/symposia/application.html>.

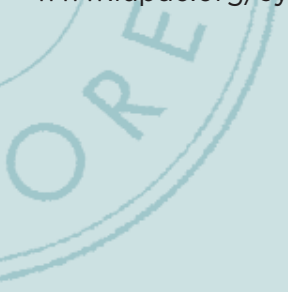

CHEMISTRY International July-August 2004 